\title{
Introduction to cancer immunotherapy
}

\section{Introduction}

Cancer immunotherapy represents the most promising new cancer treatment modality since the development of the first chemotherapies in the late 1940s.

Immunotherapy is a treatment that uses certain aspects of a person's immune system to struggle against diseases such as cancer. This can be done in two different ways:

I. Stimulating patient's immune system to work smarter to attack cancer cells.

II. Giving patient immune system components, like man-made immune system proteins.

Some types of immunotherapy are also sometimes called biologic therapy. In the last few decades immunotherapy has become an important item in the treatment of some types of cancer. The immune system is a set of organs, special cells and substances that help protect from infections and some other diseases. Immune cells and the substances circulate through the body to protect it from germs that cause infections. The immune system keeps control of all of the materials normally found in the body. Any new one that the immune system doesn't recognize raises an alarm, driving the immune system to attack it. The immune response can destroy anything containing the foreign substance, such as germs or cancer cells. However, the immune system has a more difficult time targeting cancer cells. This is because cancer starts when cells become altered and start to grow out of control. The immune system doesn't always recognize cancer cells as foreign. Cancer cells themselves can also give off substances that keep the immune system in check. Clearly, there are limits on the immune system's ability to fight cancer on its own. To overcome this, researchers have found ways to help the immune system recognize cancer cells and strengthen its response so that it will destroy them. The main types of immunotherapy now being used to treat cancer include:

\section{Monoclonal antibodies}

One way the immune system attacks foreign substances in the body is by making large numbers of antibodies. Researchers can design antibodies that specifically target a certain antigen, such as one found on cancer cells. These are known as monoclonal antibodies. To make a monoclonal antibody, researchers first have to identify the right antigen to attack. Different types of monoclonal antibodies are used in cancer treatment.

A. Naked monoclonal antibodies: Naked monoclonal antibodies are antibodies that work by themselves. There is no drug or radioactive material attached to them. These are the most common type of monoclonal antibodies used to treat cancer.

B. Naked monoclonal antibodies can work in different ways; some boost a person's immune response against cancer cells by attaching to them and acting as a marker for the body's immune system to destroy them. Some naked monoclonal antibodies boost the immune response by targeting immune system checkpoints. Other naked monoclonal antibodies work mainly by attaching
Volume 3 Issue 5 - 2015

\author{
Hajj Adel Anis \\ Cedars - Jebel Ali International Hospital, UAE
}

Correspondence: Hajj Adel Anis, Medical Oncologist at Cedars - Jebel Ali International Hospital, 9370 Rue Lajeunesse, Montreal, UAE, Tel 438-992-55I6, Email ahajj@dr.com

Received: October 25, 2015 | Published: December 07, 2015

to and blocking antigens on cancer cells, that help cancer cells grow or spread.

C. Conjugated monoclonal antibodies: Monoclonal antibodies joined to a chemotherapy drug or to radioactive particle. The monoclonal antibody is used as a homing device to take one of these substances directly to the cancer cells.

D. Bispecific monoclonal antibodies: These drugs are made up of parts of two different monoclonal antibodies, meaning they can attach to two different proteins at the same time.

\section{Immune checkpoint inhibitors to treat cancer}

An important part of the immune system is its ability to tell between normal cells in the body and those it sees as foreign. This lets the immune system attack the foreign cells while leaving the normal cells alone. To do this, it uses checkpoints -molecules on certain immune cells that need to be activated (or inactivated) to start an immune response. Cancer cells sometimes find ways to use these checkpoints to avoid being attacked by the immune system. But drugs that target these checkpoints hold a lot of promise as cancer treatments.

\section{Cancer vaccines}

We all know about vaccines given to healthy people to help prevent infections. Most cancer vaccines work the same way, but they make the person's immune system attack cancer cells. The goal is to help treat cancer or to help keep it from coming back after other treatments. But there are also some vaccines that may actually help prevent certain cancers.

\section{Non-specific cancer immunotherapies and adjuvants}

Non-specific immunotherapies don't target cancer cells specifically. They stimulate the immune system in a more general way, but this can still sometimes lead to a better immune response against cancer cells. 
These are essentially cytokines. Cytokines are chemicals made by some immune system cells. They are crucial in controlling the growth and activity of other immune system cells and blood cells. Cytokines are injected, either under the skin, into a muscle or into a vein. The problem we face, however, is that immunoselection continually occurs during tumor development with the malignant tumors that eventually emerge in patients often being resistant to elimination by the immune system. The challenge for researchers in the future is identifying patients for which immunotherapy will be efficacious and to conceive means of reactivating the immune response against tumors.

\section{Acknowledgements}

None.

\section{Conflicts of interest}

The authors declare that there is no conflict of interest. 